Cite this: J. Mater. Chem. B, 2014, 2, 3753

Received 8th February 2014 Accepted 10th April 2014

DOI: $10.1039 / c 4 t b 00208 c$

www.rsc.org/MaterialsB

\section{Characterization of carbon-coated magnetic nanoparticles using clinical blood coagulation assays: effect of PEG-functionalization and comparison to silica nanoparticles $\uparrow$}

\author{
Lukas Bircher, $\neq^{\text {ab }}$ Oliver M. Theusinger, $\$^{\mathrm{a}}$ Silvan Locher, ${ }^{a}$ Philipp Eugster, ${ }^{\text {ab }}$ \\ Birgit Roth-Z'graggen, ${ }^{\text {ab }}$ Christoph M. Schumacher, ${ }^{c}$ Jan-Dirk Studt, ${ }^{d}$ \\ Wendelin J. Stark, ${ }^{\mathrm{C}}$ Beatrice Beck-Schimmer ${ }^{\mathrm{ab}}$ and Inge K. Herrmann*ab
}

\begin{abstract}
Intravascular application of magnetic nanocarriers is a critical step in the development of new therapeutic strategies, including magnetic drug targeting or hyperthermia. However, injection of particulate matter bears the intrinsic risk of contact activation of the blood coagulation cascade. In this work, we use pointof-care assays to study coagulation dynamics and clotting parameters in blood samples exposed to relevant concentrations of surface-functionalized carbon-coated iron carbide nanomagnets using unmodified nanomagnets and poly(ethylene)glycol-functionalized nanomagnets with different endgroups, including $-\mathrm{OCH}_{3},-\mathrm{NH}_{2},-\mathrm{COOH},-\operatorname{lgG}$, and -ProteinA-protected-lgG (-IgG-ProtA). Silica nanoparticles with a comparable surface area are used as a reference material. For magnetic nanoparticles, we observe a decrease in clotting time by $25 \%$ compared to native blood at concentrations of $1 \mathrm{mg} \mathrm{mL}^{-1}$, independent of the surface functionalization, and only minor differences in receptor expression on platelets (GP-IIb-IIIa, CD62, and CD63) relative to control samples were observed. Interestingly, the inter-subject variance of the clotting time is similar to the nanoparticleinduced effect in a single subject with average clotting time. Whilst the present study is based on in vitro assays and a small group of healthy blood donors, the comparison to broadly used silica nanoparticles, and the fact that experimental intergroup variability is comparable to the observed effects from the carbon-coated nanomagnets suggests continuing investigations on their potential clinical use.
\end{abstract}

\section{Introduction}

Magnetic nanoparticles and nanoparticle-based materials offer attractive opportunities for the development of new therapeutic strategies in targeted drug delivery, magnetic separation-based blood purification, or as magnetic field-driven artificial muscles. ${ }^{\mathbf{1 - 6}}$ Nanomaterial-based approaches may revolutionize e.g., today's cancer treatment strategies by specific destruction of pathogenic cells either by targeted delivery of chemotherapeutics or by inducing hyperthermic conditions through

\footnotetext{
${ }^{a}$ Institute of Anesthesiology, University Hospital Zurich, Rämistrasse 100, CH-8091 Zurich, Switzerland. E-mail: ingekherrmann@gmail.com; Tel: +41 (0)44 2553413

${ }^{b}$ Institute of Physiology and Zurich Center for Integrative Human Physiology, University of Zurich, Winterthurerstrasse 190, CH-8057 Zurich, Switzerland

${ }^{c}$ ETH Zurich, Institute for Chemical and Bioengineering, Wolfgang-Pauli-Strasse 10, CH-8093 Zurich, Switzerland

${ }^{d}$ Division of Hematology, University Hospital Zurich, Rämistrasse 100, CH-8091 Zurich, Switzerland

$\dagger$ Electronic supplementary information (ESI) available. See DOI: 10.1039/c4tb00208c

$\$$ Denotes equal contribution to this study.
}

magnetic heating. ${ }^{7}$ The synthesis of magnetic nanoparticles with a metal core protected by a carbon shell has only recently been achieved and gives access to magnetic carriers with improved magnetic properties (saturation magnetizations up to three times higher than commonly used metal oxide particles), extending both targeting and hyperthermia applications. ${ }^{\mathbf{8}, 9}$ Although opportunities in therapy and diagnostics are numerous, detailed risk evaluations are critical in order to prevent costly downstream corrections. ${ }^{\mathbf{1 0}}$ Intravascular exposure to foreign matter, unless optimally biocompatible, induces a complex defence response of blood components, the vessel wall and other exposed tissues. ${ }^{\mathbf{1 1}}$ Injection of particulate matter, e.g. nano-sized particles (having a high specific surface area), bears the intrinsic risk of contact activation of the blood coagulation cascade (Fig. 1). ${ }^{\mathbf{1 2 - 1 4}}$ Over the recent years, it has been recognized that a range of nanoparticulate carbon-based materials influence blood coagulation and may potentially increase thrombogenic risk. ${ }^{9-15}$

Material properties, such as core material, surface morphology, charge and activity have been identified as critical parameters affecting coagulation. ${ }^{\mathbf{1 1}, 16}$ Both platelet-mediated 


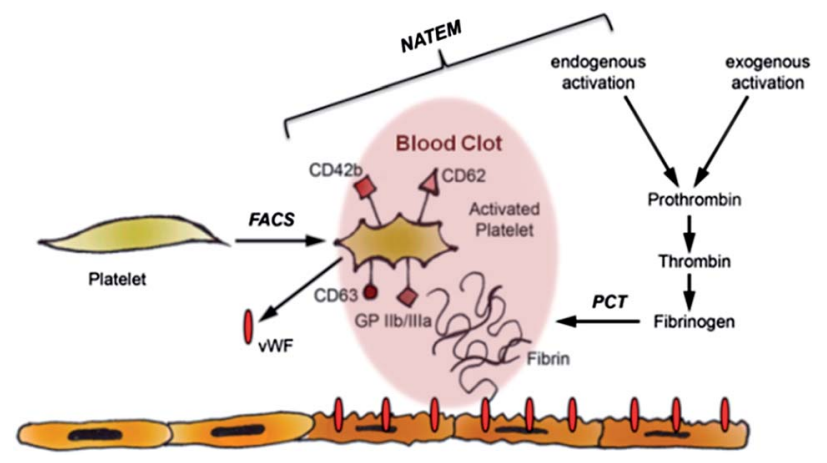

Fig. 1 Blood coagulation activation pathways including platelet activation and plasma coagulation (fibrinogen-fibrin polymerization) and corresponding diagnostic assays such as native rotational thromboelastometry (NATEM), flow cytometry (FACS) and plasma clotting time measurements (PCT).

reactions and interference in the plasma coagulation cascade, in proportions that presumably depend on the surface chemistry of the foreign material and characteristics of the blood flow, may eventually control a material's thrombogenic potential. ${ }^{17}$ The evaluation of interactions of nanomaterials with blood and the intravascular compartment has been carried out; however, development and validation of specific clinically relevant assays remains challenging.

In this work, we focus on interactions of surface-functionalized carbon-coated iron carbide magnetic nanoparticles with the blood coagulation system. The influence on coagulation dynamics, receptor expression on the platelet surface as well as plasma clotting parameters is evaluated using clinical state-ofthe-art diagnostic tests. Point-of-care assays are used to characterize coagulation behaviour, connecting the observed effect size to clinical reference range values and inter-subject variance. The influence of surface PEGylation, known to have beneficial effects on hemocompatibility and a range of different functional end groups $\left(-\mathrm{OCH}_{3}, \mathrm{NH}_{2}, \mathrm{COOH}\right.$, human $\mathrm{IgG}$, and ProteinA-protected IgG), is studied to cover different surface characteristics with regard to later applications.

\section{Results}

\section{Nanoparticle synthesis and surface functionalization}

Carbon-coated iron carbide nanoparticles were synthesized in a flame-spray pyrolysis process., ${ }^{\mathbf{8} 9}$ The combustion-derived nanomagnets have a mean diameter of $30 \mathrm{~nm}$ and a saturation magnetization of $123 \mathrm{emu} \mathrm{g}^{-1}$.,18 $^{9}$ Chemical functionalization using PEGylation agents with different end groups, including $\mathrm{OCH}_{3}, \mathrm{NH}_{2}, \mathrm{COOH}$, human IgG and ProteinA-protected IgG was confirmed by infrared spectroscopy and elemental analysis of the carbon, nitrogen and hydrogen content. PEG loading was $\sim 2 \mathrm{wt} \%$ for all functionalizations, corresponding to a calculated approximate layer thickness of $2.4 \mathrm{~nm}$, according to Rotzetter et al. ${ }^{19}$ (see ESI, Fig. S1 $\dagger$ ). Functionalized and unfunctionalized nanomagnets were then subjected to a number of established blood coagulation assays at concentrations of $0.5,1$, and $2 \mathrm{mg} \mathrm{mL}{ }^{-1}$. Amorphous silica nanoparticles with a comparable surface area were used as a reference material and saline $(\mathrm{NaCl}$ $0.9 \%$ ) served as the negative control.

\section{Protein adsorption on the nanoparticle surface}

Protein coverage of foreign surfaces is an important process during host response. Complex protein cascade systems begin to interact with a foreign surface seconds to minutes after first contact with blood. Adsorption of the most abundant proteins human serum albumin (HSA) and human fibrinogen on the nanoparticle surface was therefore quantitatively assessed using UV/VIS spectroscopy. Albumin adsorbed on unmodified nanoparticles $\left(2.5 \pm 1 \mathrm{mg} \mathrm{m}^{-2}\right.$ nanoparticle surface, see ESI, Fig. S1 $\uparrow)$, but not on PEGylated surfaces $\left(0.5 \pm 1 \mathrm{mg} \mathrm{m}^{-2}\right.$ nanoparticle surface) without influence of the functional end groups $(p<0.05)$. Compared to albumin, fibrinogen was significantly less adsorbed on the unmodified carbon surface $\left(0.7 \pm 1 \mathrm{mg} \mathrm{m}^{-2}\right.$ nanoparticle surface, $\left.p<0.05\right)$, with comparable adsorption on silica nanoparticles $\left(1.2 \pm 1 \mathrm{mg} \mathrm{m}^{-2}\right.$ nanoparticle surface for both albumin and fibrinogen).

\section{Rotational thromboelastometry (ROTEM $\left.{ }^{\circledR}\right)$}

In a whole blood coagulation assay (native thromboelastometry, NATEM), the influence of carbon-coated magnetic nanoparticles on coagulation dynamics was measured using particle concentrations of $0.5,1$ and $2 \mathrm{mg}$ per $\mathrm{mL}$ of blood. Characteristic parameters of rotational thromboelastometry include clotting time (CT), clot formation time (CFT), maximum clot firmness (MCF) and the alpha angle $(\alpha)$.

At concentrations of $0.5 \mathrm{mg} \mathrm{mL}^{-1}$, unmodified nanomagnets showed a significantly decreased CT $(p=0.001)$, while there was no significant difference observed for PEGylated particles (data not shown). At concentrations of $1 \mathrm{mg} \mathrm{mL} \mathrm{m}^{-1}$, nanomagnets significantly decreased CT (except PEG-OCH${ }_{3}$ ) by about $25 \%$, independent of functional end groups $\left(\mathrm{NH}_{2}, \mathrm{COOH}\right.$, $\mathrm{IgG}$, and IgG-ProtA) (Fig. 2a). At a concentration of $2 \mathrm{mg} \mathrm{mL}^{-1}$, a significantly decreased CT $(-30 \%)$ was found for all experimental groups (including $\mathrm{PEG}-\mathrm{OCH}_{3}$ ) (data not shown). In comparison, surface-equivalent amounts of silica nanoparticles had a particularly strong influence on CT $\left(-75 \%\right.$ at $0.5 \mathrm{mg} \mathrm{mL}^{-1}$ and $-80 \%$ at $2 \mathrm{mg} \mathrm{mL}^{-1}$ ). Only minor influences of magnetic nanoparticles on CFT were found (Fig. 2b). CFT was significantly decreased only for unmodified nanomagnets (at concentrations of $0.5-2 \mathrm{mg} \mathrm{mL}^{-1},-30 \%, p=0.002$ ) and for PEG-IgG nanomagnets at a concentration of $2 \mathrm{mg} \mathrm{mL}^{-1}(-33 \%$, $p=0.003$ ). Again, silica nanoparticles had the strongest influence and showed a decrease in CFT of $60 \%$ over the whole concentration range $(p<0.001)$. MCF and $\alpha$ were not influenced by the presence of nanomagnets, while silica significantly increased MCF and $\alpha(p<0.001)$ (Fig. $2 \mathrm{c}$ and d). All clotting parameters remained in the reference range (manufacturer's information: CT: 300-1000 s, CFT: 150-700 s; MCF: 40-65 mm; $\left.\alpha: 30-70^{\circ}\right)$, except the clotting time value for silica nanoparticles. Inter-subject variance in NATEM parameters showed a variability of $\pm 25 \%$ in CT, $\pm 15 \%$ in CFT, and below $\pm 10 \%$ in MCF and alpha $(N=6)$. No hyperfibrinolysis was observed. 
a

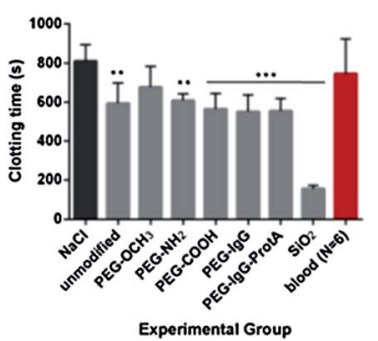

c

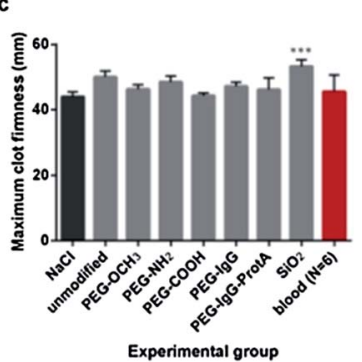

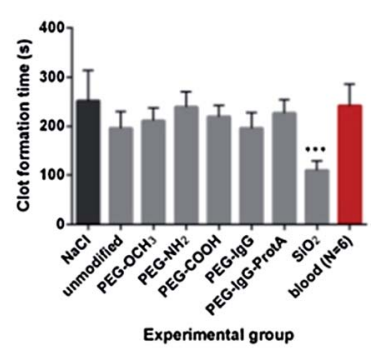

a

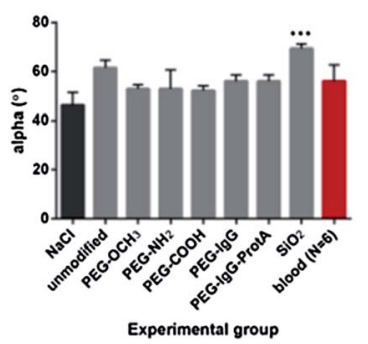

Fig. 2 Influence of surface functionalized nanomagnets ( $1 \mathrm{mg} \mathrm{mL}^{-1}, \mathrm{~N}$ $=6$ ) on characteristic parameters, i.e. clotting time $(C T, a)$, clot formation time (CFT, b), maximum clot firmness (MCF, c) and the alpha angle (alpha, d) in rotational thromboelastometry (ROTEM®-NATEM assay). Inter-subject variance was assessed by including 6 healthy volunteers (red bars). ** denotes $p<0.01$, *** means $p<0.001$.

\section{Influence on platelet receptor expression}

Platelet surface receptor expression was measured by flow cytometry analysis of whole blood samples incubated with nanoparticles at a concentration of $1 \mathrm{mg} \mathrm{mL}^{-1}$ (Fig. 3). Concentrations of $1 \mathrm{mg} \mathrm{mL}{ }^{-1}$ were chosen for further investigations as this was the minimal effect concentration. The concentration is considered to be as rather high with regard to future in vivo applications. For example, for magnetic separation based blood purification typical particle concentrations are $0.5 \mathrm{mg} \mathrm{mL}^{-1}$ or below. ${ }^{6}$

Receptor expression of activated GPIIb-IIIa (using PAC-1, recognizing only the conformationally activated GPIIb/IIIa), CD62, CD63 (markers of platelet activation targeting alpha and delta granules), CD41, CD107a, and Thrombin PAR-1 was determined in whole blood samples. CD42b was used as a platelet specific marker to identify platelets in flow cytometry. GPIIb-IIIa receptor expression was found slightly increased in samples exposed to unmodified magnetic nanoparticles while PEGylation attenuated the effect to levels comparable to the baseline. There was no significant influence of magnetic nanoparticles on the expression of CD62, CD63 (Fig. 3), CD41, CD107a, and Thrombin PAR-1 (not shown). Thrombin receptor activator for peptide 6 (TRAP) concentrations of $1 \mu \mathrm{M}, 2 \mu \mathrm{M}$ and $3 \mu \mathrm{M}$ were used as positive controls and as reference values for the observed effect sizes. The number of platelets in blood samples remained unaffected by exposure to nanomagnets, as confirmed by platelet counting.

\section{Influence on plasma clotting time}

The plasma clotting time was assessed by measuring fibrin polymerization in recalcified platelet-poor plasma at $405 \mathrm{~nm} .^{20}$

a
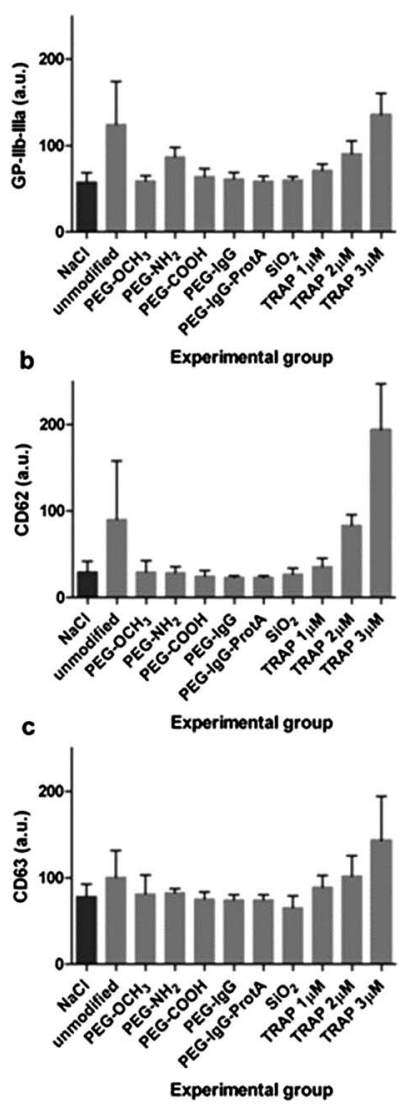

Fig. 3 Platelet receptor expression (GP-IIb-IIla (a), CD62 (b), and CD63 (c)) in response to carbon-coated magnetic and silica nanoparticles assessed by flow cytometry for particle concentrations of $1 \mathrm{mg} \mathrm{mL}^{-1}$.

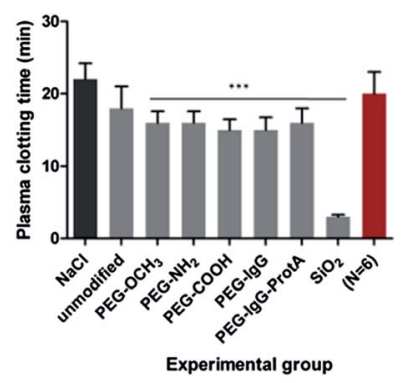

Fig. 4 Plasma clotting time measurements assessing fibrinogen polymerization in platelet-poor plasma following incubation with nanoparticles at a concentration of $1 \mathrm{mg} \mathrm{mL}^{-1}$. *** denotes $p<0.001$.

Magnetic nanoparticles had a significant influence on plasma clotting time which was shortened by approximately $20 \%$ relative to the saline controls ( $p<0.001$ ) (Fig. 4). Silica nanoparticles lead to a shortening in plasma clotting time by $80 \%(p<0.001)$.

\section{Discussion}

Carbon-coated iron carbide nanomagnets are considered to be attractive candidates for magnetic separation based blood 
purification, ${ }^{3}$ drug targeting, ${ }^{2,21}$ and hyperthermia. ${ }^{2,22}$ The coreshell geometry combines a highly magnetic metal core with stability under a wide range of conditions and stable linker attachment to the carbon surface. ${ }^{8,9,18,23}$ Surface functionalization with activity or selectivity giving agents and/or stabilizing polymers is critical for application-oriented studies, and carbon surfaces provide a unique platform for such functionalizations. However, hemocompatibility of these particles is a prerequisite for a successful implementation of nanomagnet-based strategies into therapeutic applications. ${ }^{12,24}$

Global coagulation assays showed that addition of carboncoated iron carbide nanoparticles leads to slightly decreased blood and plasma clotting times and clot formation time for concentrations of $1 \mathrm{mg} \mathrm{mL}{ }^{-1}$. In agreement with previous findings, ${ }^{12,16,20}$ PEGylation indeed reduced the observed effects on coagulation, and no significant influence of PEGylated nanomagnets on coagulation parameters was observed at concentrations of $0.5 \mathrm{mg} \mathrm{mL} \mathrm{m}^{-1}$ (Fig. 2-4). In agreement with previous work, surface PEGylation decreased protein adsorption on the particle surface, however, did not fully prevent it. ${ }^{19}$ Potential adverse effects of surface PEGylation on complement activation, as observed by Moghimi et al. ${ }^{25}$ have not been observed for the particles used in this study. ${ }^{12}$ In comparison to silica, a broadly used material in consumer products, carboncoated nanomagnets clearly showed fewer effects on blood coagulation parameters. Effects of carbon-coated nanomagnets on clotting parameters were minor and all parameters remained in the clinical reference ranges with effects on platelet receptor expression equivalent or lower to TRAP concentrations of $1 \mu \mathrm{M}$. The investigated nanoparticle concentrations of $0.5-2 \mathrm{mg} \mathrm{mL}^{-1}$, although comparatively high, are concentrations that are highly relevant in applications, such as extracorporeal magnetic separation-based blood purification processes. ${ }^{6}$ Additionally, these are typical local concentrations temporarily reached at injection sites during intravascular nanoparticle application.

In the present study, we included NATEM data of six healthy human volunteers as an internal reference allowing comparison of the observed nanoparticle-induced effect sizes with physiological inter-subject variance. Indeed, it was found that the effect of nanomagnets on clotting time of blood from a subject with average clotting time was similar to the inter-subject variance. Whereas the observed decrease in clotting time was in the order of $25 \%$ relative to the control, there were only minor differences in expression of markers of platelet activation relative to control samples. This is in good agreement with plasma clotting time measurements showing a congruent decrease in clotting time in platelet-free samples exposed to nanoparticles compared to the control. The observed effects in the global haemostasis assay can thus mainly be attributed to activation of plasmatic factors rather than platelet activation, which is in good agreement with previous studies. ${ }^{11}$ However, this approach simplifies the whole-blood coagulation by dividing it into plasma- and platelet-mediated regimes under elimination of flow conditions and blood pressure. ${ }^{17}$ While translation of results to whole-blood coagulation and in vivo conditions must be done with considerable caution, the simplification permits close examination of plasma reactions involved in thrombosis that might otherwise be obscured or greatly complicated by platelet contributions. ${ }^{17}$ Ultimately, in vivo (animal) studies are needed to clarify the significance of the observed effects (i.e. $25 \%$ reduced CT) both in healthy subjects and in subjects at risk.

\section{Conclusions}

Carbon-coated nanoparticles are potentially attractive candidates for magnetic drug delivery and blood purification. Effects of functionalized carbon-coated nanoparticles on blood coagulation were found to be minor and are expected to be of little clinical significance, especially when putting the observed effect sizes in context to the inter-subject variance. However, a safe implementation into promising therapeutic strategies requires a detailed evaluation and understanding of risks associated with exposure to the material. It is critical to carefully evaluate coagulation interactions of novel materials at an early stage to prevent late stage corrections and to identify amplifying effects and subjects at high risk. The hemocompatibility of nanomaterials is an important stepping stone in the preclinical evaluation, which heavily relies on the interpretation and translation of in vitro findings into clinics. Whereas effects of long-term exposure are challenging to evaluate with only a few models available, acute effects can be assessed using established in vitro tests. Thromboelastometry in combination with flow cytometry and fibrin polymerization assays provides an easy-to-use selection of assays to evaluate the thrombogenetic properties of nanomaterials in both a functional and mechanistic way.

This study reports a potentially useful approach to put findings into context by using state-of-the-art clinical diagnostic tests. This approach may be translatable to other nanomaterials and may become useful in the evaluation process of other nanomaterials regarding blood coagulation. Whilst the present findings are based on a small group of healthy blood donors, the comparison to broadly used silica nanoparticles, and the fact that experimental intergroup variability was comparable to the observed effects from the carboncoated nanomagnets suggests continuing investigations on their potential clinical use.

\section{Experimental}

\section{Nanoparticle synthesis and surface functionalization}

Nanoparticles were synthesized in a reducing flame spray synthesis process. ${ }^{9}$ The as-prepared nanoparticles were subjected to washing procedures in acidic aqueous solutions $(\mathrm{HCl}$, $\mathrm{pH}=2$ ) in order to remove incompletely coated metal nanoparticles. Metal leaching of the remaining particles after this treatment is negligible at neutral $\mathrm{pH}^{26}$ Surface functionalization was carried out by attaching PEGylation agents (10 kDa) with different functional end groups $\left(-\mathrm{OCH}_{3}, \mathrm{NH}_{2}, \mathrm{COOH}\right.$, and human $\operatorname{IgG}$ ) to the thiol-functionalized carbon surface of the nanomagnets using previously established procedures., ${ }^{4,12}$ In order to exclude effects of exposed IgG Fc fragments (because of the random orientation of the IgG antibody on the surface), 
ProteinA-protected PEG-IgG nanomagnets were prepared by stirring IgG-functionalized nanomagnets in a ProteinA solution. ProteinA is then expected to cap free Fc-fragments of the attached IgG antibody. The functionalized nanoparticles were characterized using transmission electron microscopy, elemental analysis and infrared spectroscopy. Amorphous silica nanoparticles (Aerosil, OX 50, Evonik, SSA: $50 \pm 15 \mathrm{~m}^{2} \mathrm{~g}^{-1}$ ) with a comparable surface area were used as a positive control. Nanoparticle dispersions were prepared by sonication of nanoparticles in saline $(0.9 \%)$.

\section{Protein adsorption on the nanoparticle surface}

Synthesized nanomagnets were characterized with regard to protein adsorption. Protein adsorption was measured using human serum albumin and fibrinogen solutions $(0.75 \mathrm{mg}$ protein per $\mathrm{mL}$ ). Nanoparticles were incubated for 15 minutes and separated from the plasma sample by magnetic separation. Absorbance of supernatants was quantified in an UV spectrometer (280 nm, GeneQuant) and compared to a reference sample (without nanoparticles). The surface concentration of protein was calculated by dividing the mass of adsorbed protein by the surface area of the exposed nanomagnets. Six or more samples were analysed per group $(N \geq 6)$.

\section{Blood collection}

Every donor signed a written consent approved by the ethical commission of Zurich. In volunteers, after having obtained written informed consent, 5 tubes of citrated blood (Vacutainer, buffered sodium citrate, $0.109 \mathrm{M}$, Belliver Industrial Estate, Plymouth, UK) were withdrawn using a $21 \mathrm{G}$ needle. The first tube was discarded to exclude coagulation activation due to vein puncture and blood withdrawal. Only volunteers who have not taken any drugs known to affect platelet function for two weeks prior to donation were included in the study. Blood hemostasis assays were carried out using a nanoparticle concentration of $0.5,1$, and $2 \mathrm{mg}$ per $\mathrm{mL}$ blood.

\section{Rotational thromboelastometry}

Rotational thromboelastometry (ROTEM® delta, TEM ${ }^{\circledR}$ International $\mathrm{GmbH}$, Munich, Germany) is a global coagulation assay for the dynamic measurement of hemostasis. ${ }^{27,28}$ Briefly, viscoelastic changes of clot initiation, formation and lysis are observed using an oscillation pin immersed in a whole blood sample. Thromboelastometry provides information on the kinetics of hemostasis: characteristic measurement parameters include the clotting time (CT), clot formation time (CFT), clot stability (maximum clot firmness, MCF) and lysis, respectively. NATEM is a ROTEM ${ }^{\circledR}$ assay which assesses clot formation, fibrin polymerisation and fibrinolysis in the absence of an activation of the clotting cascade other than recalcification and spontaneous contact activation. It provides a sensitive assessment of the dynamics of coagulation activation or inhibition.

For NATEM measurements, pre-dispersed nanomagnets or silica nanoparticles (positive control) were added to citrate blood and immediately analysed. $\mathrm{NaCl} 0.9 \%$ was used as a negative control. Six independent samples were analysed per group $(N=6)$. All ROTEM ${ }^{\circledR}$ assays were performed with reagents of the same lot number. The following parameters were determined: CT, CFT, MCF and alpha-angle $(\alpha)$. A rotational thromboelastometry device was used, equipped with four channels. Tests ran for 62 minutes. Further technical details of ROTEM® coagulation analysis can be found elsewhere. ${ }^{21}$

\section{Flow cytometry of platelet activation markers}

For flow cytometry measurements, fresh whole blood samples were exposed to nanoparticles pre-dispersed in saline. ${ }^{29} \mathrm{NaCl}$ $0.9 \%$ (vehicle) was used as a negative control. Thrombin receptor activator for peptide 6 (TRAP) was used as a positive control. The samples were exposed to nanoparticles for ten minutes at $37{ }^{\circ} \mathrm{C}$ under gentle agitation. The samples were then incubated with antibodies against glycoprotein IIb/IIIa (GPIIb/IIIa), CD41, CD62P, CD63, CD107a, and Thrombin PAR-1 for $30 \mathrm{~min}$ at $4^{\circ}$. CD42b was used to identify platelets in the flow cytometry analysis of whole blood samples. The PAC- 1 antibody recognizes the conformational change of GPIIb/IIIa on the surface of activated platelets. CD62P and CD63 are markers for alpha and delta granule after fusion with the cell membrane platelets. Samples were diluted and fixed with 1\% paraformaldehyde. All samples were analysed within two hours. Flow cytometry was performed using a BD FACSCanto ${ }^{\mathrm{TM}}$ II (Beck Dickinson, San Diego, Ca, USA). In all experiments, 10000 platelets were analysed per count. FlowJoe Version 8.8.6 was used to further analyse the data. At least three samples were analysed per group $(N \geq 3)$.

\section{Plasma clotting time assay}

Thrombin-catalyzed polymerization was monitored in re-calcified platelet poor plasma for $100 \mathrm{~min}$ as the change of turbidity at $405 \mathrm{~nm}$. Platelet-poor plasma was obtained through centrifugation, diluted with $\mathrm{NaCl}(1: 2)$ and incubated with nanomagnets ( $1 \mathrm{mg} \mathrm{mL} \mathrm{m}^{-1}$ plasma) for 5 minutes. The plasma was then recalcified through addition of calcium chloride solution (50 $\mathrm{mM}$ ) following a protocol described elsewhere. ${ }^{20}$ The plasma clotting time and the maximal velocity were recorded for each absorbance curve. At least two independent experiments were carried out each including eight samples per group.

\section{Platelet count}

A possible effect of nanomagnets on the platelet count was determined using an Advia 2120 hematology system (Siemens Healthcare).

\section{Statistics}

Bar graphs show mean \pm standard deviation values. One-way ANOVA with Bonferroni post-hoc testing was used for statistical analyses. $p<0.05$ was considered statistically significant.

\section{Author contributions}

L.B. and O.M.T. performed the thromboelastometry experiments, S.L. and P.E. performed protein adsorption and plasma 
clotting time measurements. L.B., B.R.Z., and J.D.S. analysed samples by flow cytometry, C.M.S. synthesized, functionalized and characterized the nanoparticles, J.D.S., W.J.S., B.B.S and I.K.H. designed the study, O.M.T, B.R.Z., S.L. and I.K.H. analysed the data, all of the authors participated in scientific discussions and wrote the paper.

\section{Acknowledgements}

Financial support from the Swiss National Science Foundation (NFP-64, grant no. 131268, to B.B.S. and W.J.S), the Forschungskredit of the University of Zurich (grant no. 54345802, to I.K.H.) the Hartmann-Müller Foundation (grant no. 34345825 to I.K.H.) and the Herzog-Egli Foundation (grant no. 34345824, to I.K.H.) is kindly acknowledged.

\section{Notes and references}

1 A. K. Gupta and M. Gupta, Biomaterials, 2005, 26, 3995-4021. 2 Q. A. Pankhurst, J. Connolly, S. K. Jones and J. Dobson, J. Phys. D: Appl. Phys., 2003, 36, R167-R181.

3 I. Herrmann, M. Urner, F. Koehler, M. Hasler, B. Roth Z'graggen, R. N. Grass, U. Ziegler and B. Beck-Schimmer, Small, 2010, 6, 1388-1392.

4 I. K. Herrmann, M. Urner, S. Graf, C. M. Schumacher, B. Roth-Z'graggen, M. Hasler, W. J. Stark and B. BeckSchimmer, Adv. Healthcare Mater., 2013, 2, 829-835.

5 R. Fuhrer, E. K. Athanassiou, N. A. Luechinger and W. J. Stark, Small, 2009, 5, 383-388.

6 I. K. Herrmann, A. Schlegel, R. Graf, C. M. Schumacher, N. Senn, M. Hasler, S. Gschwind, A.-M. Hirt, D. Guenther, P.-A. Clavien, W. J. Stark and B. Beck-Schimmer, Nanoscale, 2013, 5, 8718-8723.

7 S. Mornet, S. Vasseur, F. Grasset and E. Duguet, J. Mater. Chem., 2004, 14, 2161-2175.

8 R. N. Grass, E. K. Athanassiou and W. J. Stark, Angew. Chem., Int. Ed., 2007, 46, 4909-4912.

9 I. K. Herrmann, R. N. Grass, D. Mazunin and W. J. Stark, Chem. Mater., 2009, 21, 3275-3281.

10 W. J. Stark, Angew. Chem., Int. Ed., 2011, 50, 1242-1258.

11 J. Simak, in Nanotoxicity: From In Vivo and In Vitro Models to Health Risks, ed. S. C. Sahu and D. A. Casciano, John Wiley \& Sons, Ltd., 2009, p. 190.

12 I. K. Herrmann, M. Urner, M. Hasler, B. Roth-Z'Graggen, C. Aemisegger, W. Baulig, E. K. Athanassiou, S. Regenass,
W. J. Stark and B. Beck-Schimmer, Nanomedicine, 2011, 6, 1199-1213.

13 A. N. Ilinskaya and M. A. Dobrovolskaia, Nanomedicine, 2013, 8, 969-981.

14 H. Steuer, R. Krastev and N. Lembert, J. Biomed. Mater. Res. Part B, 2013, DOI: 10.1002/jbm.b.33051.

15 A. Radomski, P. Jurasz, D. Alonso-Escolano, M. Drews, M. Morandi, T. Malinski and M. W. Radomski, Br. J. Pharmacol., 2005, 146, 882-893.

16 A. Yildirim, E. Ozgur and M. Bayindir, J. Mater. Chem. B, 2013, 1, 1909-1920.

17 E. A. Vogler and C. A. Siedlecki, Biomaterials, 2009, 30, 18571869.

18 C. M. Schumacher, I. K. Herrmann, S. B. Bubenhofer, S. Gschwind, A.-M. Hirt, B. Beck-Schimmer, D. Günther and W. J. Stark, Adv. Funct. Mater., 2013, 23, 4888-4896.

19 A. C. C. Rotzetter, C. M. Schumacher, T. Zako, W. J. Stark and M. Maeda, Langmuir, 2013, 29, 14117-14123.

20 R. Tavano, D. Segat, E. Reddi, J. Kos, M. Rojnik, P. Kocbek, S. Iratni, D. Scheglmann, M. Colucci, I. M. Echevarria, F. Selvestrel, F. Mancin and E. Papini, Nanomedicine, 2010, 5, 881-896.

21 C. Alexiou, W. Arnold, R. J. Klein, F. G. Parak, P. Hulin, C. Bergemann, W. Erhardt, S. Wagenpfeil and A. S. Lubbe, Cancer Res., 2000, 60, 6641-6648.

22 A. Jordan, R. Scholz, P. Wust, H. Fahling, J. Krause, W. Wlodarczyk, B. Sander, T. Vogl and R. Felix, Int. J. Hyperthermia, 1997, 13, 587-605.

23 I. K. Herrmann, R. N. Grass and W. J. Stark, Nanomedicine, 2009, 4, 787-798.

24 A. N. Ilinskaya and M. A. Dobrovolskaia, Nanomedicine, 2013, 8, 773-784.

25 S. M. Moghimi, A. J. Andersen, S. H. Hashemi, B. Lettiero, D. Ahmadvand, A. C. Hunter, T. L. Andresen, I. Hamad and J. Szebeni, J. Control. Release, 2010, 146, 175-181.

26 M. Rossier, F. M. Koehler, E. K. Athanassiou, R. N. Grass, M. Waelle, K. Birbaum, D. Günther and W. J. Stark, Ind. Eng. Chem. Res., 2010, 49, 9355-9362.

27 A. Straub, D. Schiebold, H. P. Wendel, C. Hamilton, T. Wagner, E. Schmid, K. Dietz and G. Ziemer, Eur. J. Cardio-Thorac. Surg., 2008, 34, 641-647.

28 O. M. Theusinger, J. Nurnberg, L. M. Asmis, B. Seifert and D. R. Spahn, Eur. J. Cardio-Thorac. Surg., 2010, 37, 677-683. 29 A. D. Michelson, M. R. Barnard, L. A. Krueger, A. L. Frelinger Iii and M. I. Furman, Methods, 2000, 21, 259-270. 